\title{
Melanose neurocutânea. Relato de caso com melanoma maligno do sistema nervoso central
}

\author{
J.M. J uang, A.C.L.G. Silva, M.C. Pires, N.Y.S. Valente, J .A.S. Sittart \\ Serviço de Dermatologia do Hospital do Servidor Público Estadual de São Paulo, São Paulo, SP.
}

RESUMO. A melanose neurocutânea é uma síndrome rara caracterizada por nevos melanocíticos congênitos gigantes e excessi va melanose da leptomeninge. A síndrome parece representar um erro na morfogênese do neuroectoderma embrionário. Os autores apresentam o caso e necropsia de um

\section{INTRODUÇÃO}

A melanose neurocutânea (MNC) éuma síndrome congênita rara caracterizada pel o desenvol vimento de nevos melanocíticos congênitos e neoplasias melanocíticas benignas ou malignas do sistema nervoso central. Foi descrita, em 1861, por Rokitansky, e o termo usado pela primeira vez, em 1948, por Bogaert. Até então, foram relatados 97 casos, a grande maioria em revistas médicas de Neurocirurgia.

\section{RELATO DO CASO}

Paciente de 19 anos, masculino, negro, natural e procedente de São Paulo. Ao nascimento apresentava nevo mel anocíti co pi l oso gi gante em "cal ção de banho" e múltipl os nevos spilus nos membros superiores, inferiores e tronco (figs. 1, 2 e 3). A dois meses da internação, teve início intensa cefaléia e crises convulsivas. Deu entrada no Hospital do Servidor Público Estadual de São Paulo em estado geral comprometido e emagrecido; as crises convulsivas tornaram-setônico-clônicas generalizadas e refratárias ao tratamento. Evoluiu com deterioração do nível da consciência, coma e óbito por sepsis.

\section{Diagnósticos dermatológi cos}

Nevo melanocítico gigante piloso com morfotopografia tipo "calção de banho" e nevos spilus múltiplos. paciente masculino de 19 anos que desenvolveu melanoma maligno do sistema nervoso central.

UnITERMOS: Melanose neurocutânea. Melanoma. Nevos congênitos.

\section{EXAMES COMPLEMENTARES}

Exame do líquor: leucócitos: $6 / \mathrm{mm}^{3}$; linfócitos: 45/ $\mathrm{mm}^{3}$; monócitos: $13 / \mathrm{mm}^{3}$ e eosinófilos: $1 / \mathrm{mm}^{3}$. Hemácias: 0. Proteínas: $510 \mathrm{mg} / 100 \mathrm{~mL}$. Glicose: 99mg/100mL. Cloretos: $7 \mathrm{mg} / 100 \mathrm{~mL}$. U réia: $18 \mathrm{mg} /$ 100mL. Reação de Pandy: ++. Presença de células com atipia e pigmentação citoplasmática acastanhada sugestiva de melanina. Cultura negativa. Ressonância magnética: contrastação leptomeníngea e ependimária difusa e grave, massa frontal parassagital esquerda. Anti-HIV: negativo.

\section{Necropsia}

Grande nevo congêni to estendendo-se por todo o abdome, dorso e raízes dos membros inferiores até metade da coxa.

Múltiplos nevos melanocíticos intradérmicos, sem atipia na cabeça, tórax e membros (fig. 4).

Melanose leptomeníngea.

Melanoma maligno na região frontal parassagital esquerda (fig. 5). Broncopneumonia.

\section{DISCUSSÃo}

A MNC ocorre mais freqüentemente em brancos, mas também já foi descrita em negros, podendo-se apresentar em natimortos, neonatos e, ocasionalmente, na segunda década de vida, como no caso descrito. A relação mulheres/homens é de 1:2 e a história familiar para nevos melanocíticos múltiplos já foi relatada2,4. 


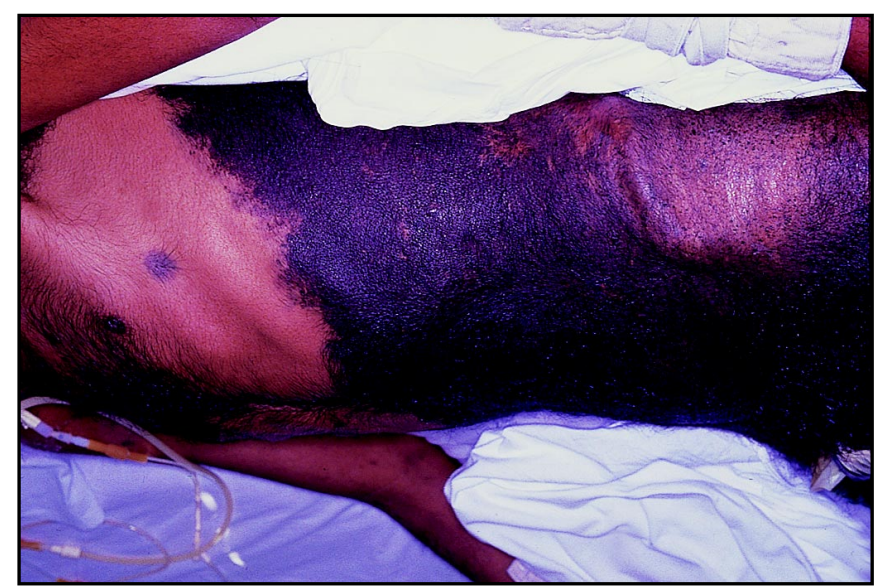

Fig. 1 - N evo mel anocítico gigante.

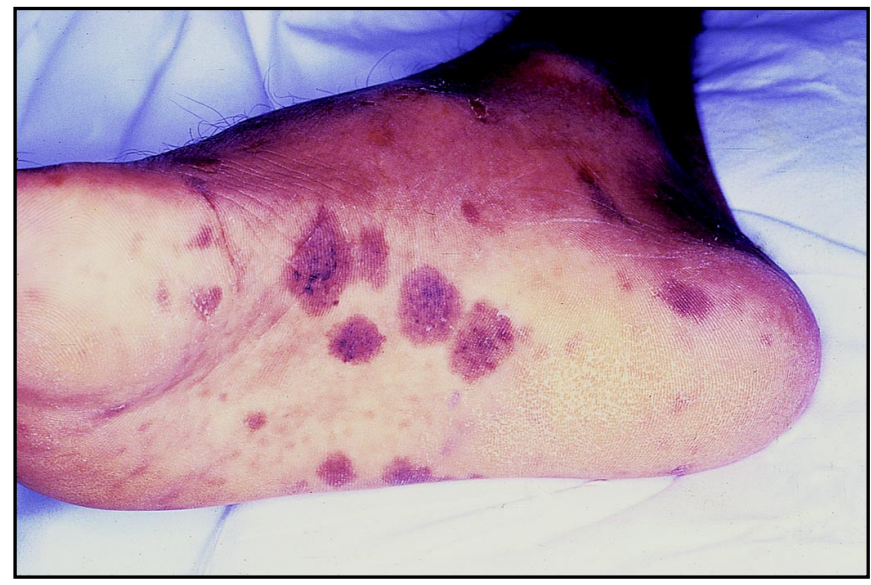

Fig. 3 - Nevos spilus no pé.

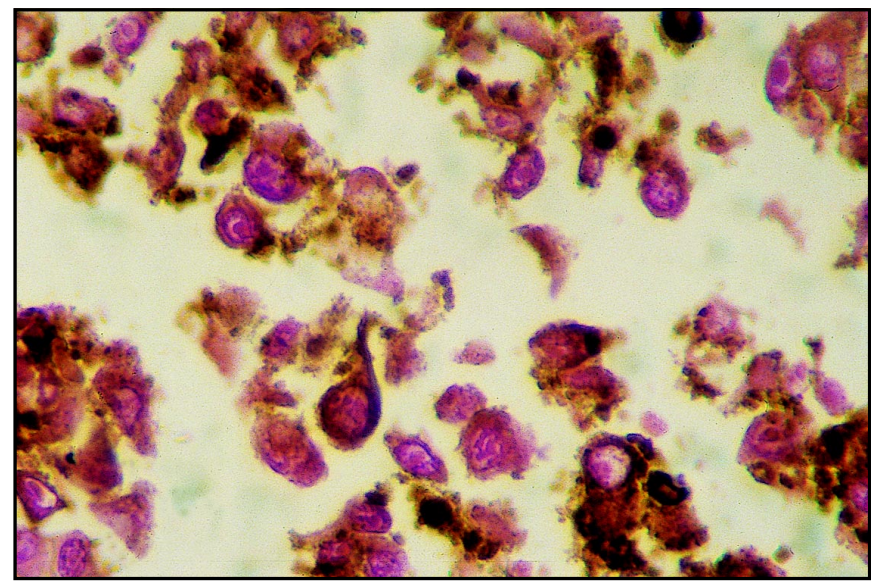

Fig. 5 - M el anócitos atípi cos no si stema nervoso central.

Nos dados clínicos, em $66 \%$ dos casos são encontradas lesões pigmentadas gigantes, a mai oria com distribui ção em "cal ção de banho" ou lombossacral . E m pacientes com lesões na cabeça ou pescoço, 94\% desenvolvem MNC, dos quais $62 \%$ apresentam melanoma de leptomeninge com localização mais freqüente nos lobos frontal e temporal.

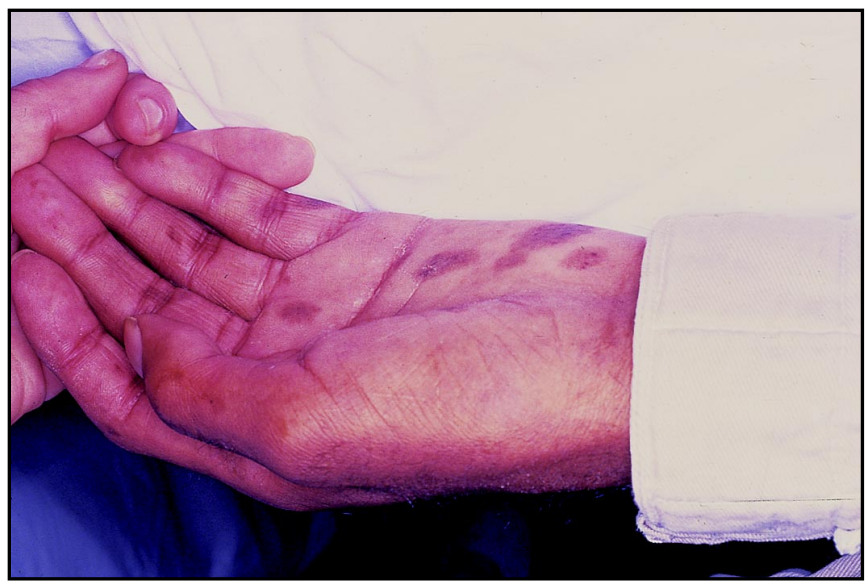

Fig. 2 - Nevos spilus na mão.

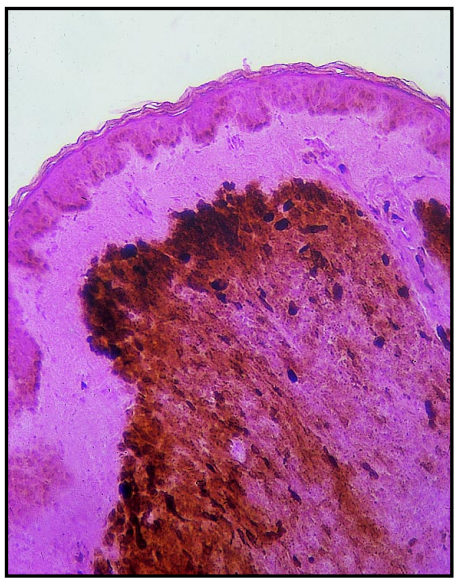

Fig. 4 - Aspecto histopatológico do nevo melanocítico intradérmico.

As manifestações neurológicas ocorrem, principalmente, nos dois primeiros anos de vida, com sinais e sintomas de hipertensão intracraniana.

$\mathrm{Na}$ histopatologia, 2/3 das lesões são nevos melanocíticos intradérmicos e $1 / 3$ nevos compostos $^{2,3}$. As células névicas estendem-se à derme reticular e, ocasionalmente, até a hipoderme.

E m 1972, F ox propôs critérios diagnósticos para a MNC que foram revisados recentemente ${ }^{1-3}$.

\section{Critérios diagnósticos da MNC}

1) Nevos congênitos múltiplos ou grandes em associação com melanose ou melanoma das meninges. Os nevos devem ter mais que $20 \mathrm{~cm}$ de diâmetro em adultos. Em neonatos e crianças: $9 \mathrm{~cm}$ na cabeça ou $6 \mathrm{~cm}$ no corpo. Múltiplos se referindo a três ou mais lesões.

2) Sem evidência de melanoma cutâneo, exceto em pacientes cujas lesões meníngeas examinadas sejam histol ogicamente benignas.

3) Sem evidência de melanoma meníngeo, exceto em pacientes cujas lesões cutâneas examinadas sejam histol ogicamente benignas. 
Os casos com confirmação histológica de lesões do SNC são considerados definitivos, enquanto os outros são ditos provisórios.

Encontra-se, também, associado à MNC: divertícul o de $\mathrm{Meckel}^{5}$, anormalidades do trato urinário, como cistos renais e neoplasias; lipoma intra-espinhal ${ }^{6}$, meningioma e cisto aracnóide melanótico intra-espinhal ${ }^{7}$ e hipoplasia do vermis inferior ${ }^{8}$. A infecção das meninges, assim como do trato respiratório, são comuns e fatais.

E m relação ao tratamento e prognóstico, $70 \%$ dos doentes morrem antes dos dez anos de vida ${ }^{1,2}$ por malignização ou hidrocefalia ${ }^{9-11}$. Infelizmente, a terapia antineoplásica não melhora os sintomas e não altera o curso rápido da MNC. As medidas cirúrgicas paliativas, tais como o shunt para a diminuição da pressão intracraniana, promovem melhora transitória. Em relação a cirurgia profilática dos nevos, muitos autores a advogam, já que o risco de desenvolvimento de melanoma se situa na faixa de $5 \%$ a $15 \%$, porém, no caso da MNC sintomática, raramente é uma indicação.

\section{CONCLUSÃO}

Apresentamos o caso por sua raridade. O acompanhamento desses pacientes deve ser feito com freqüência para se ter diagnósti co precoce e melhora da quali dade de vida, já que a evol ução da doença é fatal.

\section{SUMMARY}

Neurocutaneous melanosis. Case report of a malignant melanoma of the central nervous system

The neurocutaneous melanosis is a rare syndrome in which the congenital melanocytic nevi and excessi vemel anosis are the main features. The syndrome seems to be a mor phogenesi s error of the embryonic neuroectoderm. A clinical case with necroscopy in a 19 year-old man who had developed malign melanoma in his central nervous system is reported. [Rev Ass Med Brasil 1998; 44(1): 50-2.]

KEY WORDS: Neurocutaneous melanosis. Melanoma. Congenital naevus.

\section{REFERÊ NCIAS BIBLIOGRÁFICAS}

1. Sandsmark M, Eskeland G, Skullerud $K$ et al. Neurocutaneous melanosis. Case report and a brief review. Scand J Plast Reconstr Hand Surg 1993; 28: 150-4.

2. Fox H, Emery J L, Goodbody RA, Yates P.O. Neurocutaneous melanosis. Arch Dis Child 1964; 39: 508-16.

3. Kadonaga J N, Frienden IJ. Neurocutaneous melanosis. Definition and review of the literature. J Am Acad Dermatol 1991; 24: 747-55.

4. Willshaw L, Pérez MF, L cassie YS. Melanosis neurocutanea familiar. Rev Chil Ped 1990; 53(5): 443-6.

5. MacLachlan WWG. Extensive pigmentation of the brain associated with nevi pigmentosi of the skin. J Med Res 1913; 29: 433-7.

6. Heuzen EP, Kaiser MC, Sleglete RGM. Neurocutaneous melanosis associated with intraspinal lipoma. Neuroradiology 1989; 31: 349-51.

7. Kasantikul V, Shuangshoti S, Pattanaruenglai $A$ et al. Intraspinal melanotic arachnoid cyst and lipoma in neurocutaneous melanosis. Surg Neurol 1989; 31: 13841.

8. Ko SF, Wang H S, Lui TN et al. Neurocutaneous melanosis associated with inferior vermian hypoplasia: MR findings. J Comput Assist Tomogr 193; 17(5): 691-5.

9. Coutinho LMB, Coutinho MF, Teixeira LC et al. Melanose neurocutânea. Estudo autópsico de um caso. Arq Neuro Psiq 1985; 43(1): 73-80.

10. Zarragoitia OL, Cruz AL, Iglesias DG. Melanosis neurocutanea. Presentacion clinica de un paciente. Rev Cubana Ped 1989; 61(1): 81-5.

11. Lamas E, Lobato RD, Sotelo T et al. Neurocutaneous melanosis. Report of a case and review of the literature. Acta Neurochir 1977; 36: 93-105. 